\title{
Mathematical Modeling of Effect of Pumping Rate on Contaminant Transport in Riverbank Filtration System
}

\section{1*ABUBAKAR, AD; ${ }^{2}$ OLAYIWOLA, RO $;{ }^{2}$ MOHAMMED, AA ; ${ }^{2}$ COLE, AT}

\author{
${ }^{*}$ Department of Mathematics, Federal University Gashu'a, Yobe State, Nigeria \\ ${ }^{2}$ Department of Mathematics, Federal University of Technology, Minna, Nigeria \\ *Corresponding Author Email: ahmeddauda8@ gmail.com; Tel: 07068233938
}

\begin{abstract}
Riverbank filtration (RBF) is a natural technology that is used for river water treatment. This research seeks to investigate the effect of pumping rate on the transport of colloids in RBF. However, this work considered Dissolved Organic Matter (DOM) as a nutrient for bacteria. The mathematical model consists of groundwater flow equation and colloids concentration equations. The equations were solved analytically using parameter expanding method and Eigen function expansion techniques. The results obtained are presented graphically and discussed. It was observed that increase in pumping rate value enhance both the hydraulic head and concentration of colloids which slightly reduces the quality of pumped water from RBF.
\end{abstract}

DOI: $\underline{\text { https://dx.doi.org/10.4314/jasem.v25i2.10 }}$

Copyright: Copyright (C) 2021 Abubakar et al. This is an open access article distributed under the Creative Commons Attribution License (CCL), which permits unrestricted use, distribution, and reproduction in any medium, provided the original work is properly cited.

Dates: Received: 12 December 2020; Revised: 26 January 2021; Accepted: 12 February 2021

Keywords: Riverbank filtration, analytical model, colloids, hydraulic head and pumping rate

Riverbank filtration (RBF) can be defined as the natural or induced transport of water from the river to the aquifer, through the riverbed. In 1800, this system was initially reported in United Kingdom, and is now widely accepted in Europe. In Germany, $63 \%$ of fresh water sources are from groundwater while $15.3 \%$ are from RBF and groundwater recharge. In Netherlands, $39 \%$ of surface water is treated using RBF and/or dune recharge (Ray, 2011). The effectiveness and efficiency of riverbank filtration largely depend on local conditions which include the geochemistry of water (from the aquifer and river), the hydrogeology and hydrology of the site, the geochemistry of microbial populations, and associated metabolic activities (Lee and Lee, 2010). Other factors are soil texture and quality (Mustafa et al., 2014). The overall design of RBF systems requires detailed hydrogeological site investigation, knowledge about the hydrological characteristics of the catchment as well as defining the catchment area (Grischek et al., 2002). The effect of hydraulic conductivity on one dimensional contaminant transport in RBF system was investigated by Mustafa et al. (2018) using Green's function approach. The result obtained show that increase in hydraulic conductivity value led to an increase in contaminant concentration in pumping well area. Kim et al. (2003) investigated numerically the effect of dissolve organic matter and bacteria on contaminant transport in riverbank filtration. The results show that contaminant transport is enhanced markedly in the presence of DOM and bacteria, and the impact of DOM on contaminant mobility is greater than that of bacteria under examined conditions. Mustafa et al. (2014) reviewed that there are few analytical models of RBF system. From their investigation they categorized the available models into two: the first category describes the flow of groundwater induced from wells near the river. While the second category describes the pollutants transportation from river to the well. They also concluded that the analytical model that simulate the potential of microorganism in RBF systems are very rear. Shamsuddin et al. (2014) presented a case study of bank infiltration (BI) method which evaluates the effects of groundwater pumping and BI operation on the installation wells as well determine the effects of pumping rate on flow paths, travel time, the size of the pumping and capture zone delineation. The results indicate that the migration of river water into the aquifer is generally slow and depends on the pumping rate and distance from well to the river. Singh et al. (2011) conducted a comparative study between Laplace Transform Technique and Fourier Transform Technique in solving onedimensional transport equation along unsteady groundwater flow in semi-infinite aquifer. Their result indicates that Fourier Transform Technique is better 
than Laplace Transform Technique in predicting contaminants concentration along groundwater flow in semi homogenous aquifer. Aiyesimi and Jimoh (2012) used computational analysis to study one-dimensional non-linear reactive contaminant flow with an initial continuous point source. They discovered that from the origin, the concentration decreases with increase in time and distance. Chattopadhyay and Deo (2018) discussed various mathematical models involving water pollutant transport equation in Damoda River, Bermo Region. The implicit central scheme in space and a forward difference method in time was also given to evaluate the generalized transport equation. Different parameters were varied and they observed a general decrease in contaminant concentration. Bohaienko and Bulavatsky (2019) developed a mathematical model of solutes migration under the conditions of groundwater filtration with $\mathrm{k}-$ Caputo fractional derivatives. They use finite difference scheme to simulate the dynamics of anomalous soluble substance migration under the conditions of twodimensional steady-state plane-vertical filtration with a free surface. The results of numerical experiments on modeling the dynamics of the considered processes were presented. Gothwal and Thatikonda (2017) studied a mathematical model for the transport of fluoroquinolone and its resistant bacteria in aquatic environment. The simulation results of the model for different cases show that the concentration of

$$
S_{s} \frac{\partial h}{\partial t}=K \frac{\partial^{2} h}{\partial x^{2}}-\frac{q}{Q_{o}\left(1-\frac{x}{L+x}\right)} S_{s} e^{-\frac{\beta}{R_{c}} t}+\lambda_{0} C_{c}
$$

antibiotic, organic matter, segregation rate, and horizontal transfer rate are the governing factors in the variation of population density of resistant bacteria. In this present work, we seek to investigate the effect of variable dependent pumping rate on the concentration of the colloids using parameter expanding method and Eigen function expansion techniques.

Mathematical Formulation: We consider a situation where contaminants are present together with dissolve organic matter (DOM), bacteria and virus in an aquifer under simplistic river bank filtration conditions. The aquifer is conceptualized as a five-phase system: three mobile colloidal phases, an aqueous phase and a stationary solid phase. An analytical approach is used to describe the interactions of contaminants with DOM, bacteria, virus and solid matrix. It is assumed that the aquifer is saturated with dissolve organic matter and DOM is utilized as a nutrient for bacteria. The aquifer is unconfined, homogeneous and isotropic. The pumping rate of the pumping well is assumed to depend on the space variable $\mathcal{X}$. Based on the above assumptions, the equations governing the phenomenon are as follows:

The one-dimensional groundwater flow equation in aquifer is given as:

The mass balance equation for bacteria (captured and suspended) in the aqueous phase of saturated porous media may be describe as:

$$
\begin{aligned}
& \varepsilon \frac{\partial C_{b}}{\partial t}+\rho_{b} K_{b} \frac{\partial C_{b}}{\partial t}-\varepsilon K \frac{\partial h}{\partial x} \frac{\partial C_{b}}{\partial x}=\varepsilon \frac{\partial}{\partial x}\left(D_{b} \frac{\partial C_{b}}{\partial x}\right)+\frac{\mu_{\max }}{K_{s}}\left(\begin{array}{l}
C_{c}+\frac{\rho_{s} K_{1} C_{c}}{\varepsilon}+C_{o} K_{2} C_{c}+ \\
C_{b} K_{3} C_{c}+C_{v} K_{5} C_{c}
\end{array}\right) \varepsilon C_{b}- \\
& K_{d m b} \varepsilon C_{b}+K_{o} Y_{b} \varepsilon C_{o}+\frac{\mu_{\max }}{K_{s}}\left(\begin{array}{l}
C_{c}+\frac{\rho_{s} K_{1} C_{c}}{\varepsilon}+\frac{\rho_{b} K_{b} C_{b} K_{4} C_{c}}{\varepsilon}+ \\
\frac{\rho_{v} K_{v} C_{v} K_{6} C_{c}}{\varepsilon}
\end{array}\right) \rho_{b} K_{b} C_{b}-K_{d i b} \rho_{b} K_{b} C_{b}
\end{aligned}
$$

The mass balance equation for virus (captured and suspended) in the aqueous phase of saturated porous media may be describe as:

$$
\begin{aligned}
& \varepsilon \frac{\partial C_{v}}{\partial t}+\rho_{v} K_{v} \frac{\partial C_{v}}{\partial t}-\varepsilon K \frac{\partial h}{\partial x} \frac{\partial C_{v}}{\partial x}=\varepsilon \frac{\partial}{\partial x}\left(D_{v} \frac{\partial C_{v}}{\partial x}\right)+\frac{\mu_{\max }}{K_{s}}\left(\begin{array}{l}
C_{c}+\frac{\rho_{s} K_{1} C_{c}}{\varepsilon}+C_{o} K_{2} C_{c}+ \\
C_{b} K_{3} C_{c}+C_{v} K_{5} C_{c}
\end{array}\right) \varepsilon C_{v} \\
& -K_{d m v} \varepsilon C_{v}+\frac{\mu_{\max }}{K_{s}}\left(C_{c}+\frac{\rho_{s} K_{1} C_{c}}{\varepsilon}+\frac{\rho_{b} K_{b} C_{b} K_{4} C_{c}}{\varepsilon}+\frac{\rho_{v} K_{v} C_{v} K_{6} C_{c}}{\varepsilon}\right) \rho_{v} K_{v} C_{v}-K_{d i v} \rho_{v} K_{v} C_{v}
\end{aligned}
$$

The mass balance equation for contaminant may be expressed as: 


$$
\begin{aligned}
& R_{c} \frac{\partial C_{c}}{\partial t}-K \frac{\partial h}{\partial x} \frac{\partial C_{c}}{\partial x}=\frac{\partial}{\partial x}\left(D_{c} \frac{\partial C_{c}}{\partial x}\right)-\frac{\mu_{\max } C_{b}}{K_{s} Y_{b}}\left(1+\rho_{s} K_{1}+\rho_{b} K_{b}\right) C_{c}- \\
& \frac{\mu_{\max } C_{v}}{K_{s} Y_{v}}\left(1+\rho_{s} K_{1}+\rho_{v} K_{v}\right) C_{c}+\frac{q}{Q_{o}\left(1-\frac{x}{L+x}\right)} S_{s} e^{-\frac{\beta}{R_{c}} t}
\end{aligned}
$$

The mass balance equation for DOM in the aqueous phase may be expressed as:

$\varepsilon \frac{\partial C_{o}}{\partial t}+\rho_{s} K_{0} \frac{\partial C_{o}}{\partial t}-\varepsilon K \frac{\partial h}{\partial x} \frac{\partial C_{o}}{\partial x}=\varepsilon \frac{\partial}{\partial x}\left(D_{o} \frac{\partial C_{o}}{\partial x}\right)-\frac{\mu_{\max } C_{o} K_{2} C_{c o}}{K_{s} Y_{b}} \varepsilon C_{b}$

Where:

$h(x, t)$ is the hydraulic head, $H$ is the head value at $t=0, Q=\lambda_{o} C_{c}$ is the volumetric flux per unit volume representing source or sink terms/reaction rate (concentration of contaminant) in the source or sink, $\beta$ is the biological processes at the riverbed, $R_{c}$ is the retardation factor, $q$ is the stream depletion flow rate, $S_{S}$ is the specific storage coefficient, $K$ is the hydraulic conductivities,

$m_{o}(x, t)$ is the concentration of contaminant measured at the pumping well, $Q_{o}$ is the pumping rate,

$L$ is the distance of the pumping well from the river, $C_{b}, C_{v}, C_{c} \& C_{o}$ are the concentration of the bacteria, virus, contaminant and DOM suspended in the aqueous phase respectively, $\varepsilon$ is the water content, $V_{p}$ is the pore water velocity, $D_{b}, D_{v}, D_{c} \& D_{o}$ are the hydrodynamic dispersion coefficient of bacteria, virus, contaminant and DOM respectively, $\rho_{b} \& \rho_{v}$ are the density of bacteria and virus respectively, $\sigma_{b} \& \sigma_{v}$ are the volumetric fraction of bacteria and virus respectively, $K_{b}, K_{v}$ are the linear equilibrium distribution coefficient of bacteria and virus between the aqueous phase and the solid phase respectively, $K_{0}$ is the first-order decay rate coefficient of DOM, $K_{s}$ is the half saturation constant, $Y_{b}$ and $Y_{v}$ is the yield coefficient of bacteria and virus, $\mu_{\max }$ is the maximum growth rate, $K_{1}$ is the linear equilibrium distribution coefficient of contaminants between the aqueous phase and the solid matrix, $K_{2}$ is the linear equilibrium distribution coefficient of contaminants between the aqueous phase and DOM, $K_{3} \& K_{4}$ are the linear equilibrium distribution coefficient of contaminants between the aqueous phase, mobile and immobile bacteria respectively, $K_{5} \& K_{6}$ are the linear equilibrium distribution coefficient of contaminants between the aqueous phase, mobile and immobile virus respectively, $K_{d m b} \& K_{d i b}$ are the decay rate coefficient of mobile and immobile bacteria respectively, $K_{d m v} \& K_{d i v}$ are the decay rate coefficient of mobile and immobile virus respectively, $\rho_{s}$ is the dry bulk density of solid matrix, $\sigma_{c s}$ is the mass fraction of contaminants sorbed on solid matrix, $Q_{o c}$ is the utilization rate of contaminants sorbed on DOM, $\sigma_{c o}$ is the mass fraction of contaminants attached to DOM,

The dependence of diffusion coefficients on the concentration of contaminant is taken in to account by the mathematical expressions:

$$
\begin{aligned}
& D_{b}=D_{b o} e^{\sigma C_{c}}, D_{v}=D_{v o} e^{\eta C_{c}}, \quad D_{c}=D_{c o} e^{v C_{c}} \\
& \text { and } \quad D_{o}=D_{o o} e^{\mu C_{c}}
\end{aligned}
$$

The initial and boundary conditions associated with the equations are formulated as: 


$$
\left.\begin{array}{ccc}
h(x, 0)=H, & h(0, t)=H, & \left.\frac{\partial h}{\partial x}\right|_{x=L}=0 \\
C_{b}(x, 0)=\frac{C_{b 0} x}{L}, & -\left.D_{b}^{*} \frac{\partial C_{b}}{\partial x}\right|_{x=0}+C_{b}(0, t)=0, & \left.\frac{\partial C_{b}}{\partial x}\right|_{x=L}=0 \\
C_{v}(x, 0)=\frac{C_{v 0} x}{L}, & -\left.D_{v}^{*} \frac{\partial C_{v}}{\partial x}\right|_{x=0}+C_{v}(0, t)=0, & \left.\frac{\partial C_{v}}{\partial x}\right|_{x=L}=0 \\
C_{c}(x, 0)=\frac{C_{c 0} x}{L}, & -\left.D_{c}^{*} \frac{\partial C_{c}}{\partial x}\right|_{x=0}+C_{c}(0, t)=0, & \left.\frac{\partial C_{c}}{\partial x}\right|_{x=L}=0 \\
C_{o}(x, 0)=\frac{C_{o 0} x}{L}, & -\left.D_{o}^{*} \frac{\partial C_{o}}{\partial x}\right|_{x=0}+C_{o}(0, t)=0, & \left.\frac{\partial C_{o}}{\partial x}\right|^{=0}
\end{array}\right\}
$$

Method of Solution: Dimensional analysis: Equation (1) - (7) were non-dimensionalized using the following dimensionless variables

$$
t^{\prime}=\frac{K t}{S_{s} L^{2}}, \quad h^{\prime}=\frac{h}{H}, \quad x^{\prime}=\frac{x}{L}, \quad \phi=\frac{C_{b}}{C_{b o}}, \quad \psi=\frac{C_{v}}{C_{v o}}, \quad \theta=\frac{C_{c}}{C_{c o}}, \quad \varphi=\frac{C_{o}}{C_{o o}}
$$

Then we obtain

$$
\begin{aligned}
& \frac{\partial h}{\partial t}=\frac{\partial^{2} h}{\partial x^{2}}-\frac{\lambda_{1}}{\left(1-\frac{x}{1+x}\right)} e^{-\lambda_{3} t}+\lambda_{2} \theta \\
& R_{b} \frac{\partial \phi}{\partial t}-\alpha_{0} \frac{\partial h}{\partial x} \frac{\partial \phi}{\partial x}=D_{1} \frac{\partial}{\partial x}\left(\left(1+\sigma_{1} \theta\right) \frac{\partial \phi}{\partial x}\right)-\alpha_{1} \phi+\alpha_{4}\left(\alpha_{5} \theta+\alpha_{6} \varphi \theta+\alpha_{7} \phi \theta+\alpha_{8} \psi \theta\right) \phi+ \\
& \alpha_{9}\left(\alpha_{5} \theta+\beta_{1} \phi \theta+\beta_{2} \psi \theta\right) \phi+\alpha_{3} \varphi \\
& R_{v} \frac{\partial \psi}{\partial t}-\alpha_{0} \frac{\partial h}{\partial x} \frac{\partial \psi}{\partial x}=D_{2} \frac{\partial}{\partial x}\left(\left(1+\eta_{1} \theta\right) \frac{\partial \psi}{\partial x}\right)-r_{0} \psi+r_{1}\left(\alpha_{5} \theta+\alpha_{6} \varphi \theta+\alpha_{7} \phi \theta+\alpha_{8} \psi \theta\right) \psi \\
& +r_{2}\left(\alpha_{5} \theta+\beta_{1} \phi \theta+\beta_{2} \psi \theta\right) \psi \\
& R_{c} \frac{\partial \theta}{\partial t}-\alpha_{\mathrm{o}} \frac{\partial h}{\partial x} \frac{\partial \theta}{\partial x}=D_{3} \frac{\partial}{\partial x}\left(\left(1+v_{1} \theta\right) \frac{\partial \theta}{\partial x}\right)-m_{1} \phi \theta-m_{2} \psi \theta+\frac{m_{3}}{\left(1-\frac{x}{1+x}\right)} e^{-\lambda_{3} t} \\
& R_{o} \frac{\partial \varphi}{\partial t}-\alpha_{0} \frac{\partial h}{\partial x} \frac{\partial \varphi}{\partial x}=D_{4} \frac{\partial}{\partial x}\left(\left(1+\mu_{1} \theta\right) \frac{\partial \varphi}{\partial x}\right)-g_{1} \phi \varphi \theta \\
& h(x, 0)=1, \quad h(0, t)=1,\left.\quad \frac{\partial h}{\partial x}\right|_{x=1}=0 \\
& \phi(x, 0)=x, \quad-\left.D_{b}^{*} \frac{\partial \phi}{\partial x}\right|_{x=0}+\phi(\mathrm{O}, t)=\mathrm{O},\left.\quad \frac{\partial \phi}{\partial x}\right|_{x=1}=\mathrm{O} \\
& \left.\psi(x, 0)=x, \quad-\left.D_{v}^{*} \frac{\partial \psi}{\partial x}\right|_{x=0}+\psi(0, t)=0,\left.\quad \frac{\partial \psi}{\partial x}\right|_{x=1}=0\right\} \\
& \theta(x, 0)=x, \quad-\left.D_{c}^{*} \frac{\partial \theta}{\partial x}\right|_{x=0}+\theta(0, t)=0,\left.\quad \frac{\partial \theta}{\partial x}\right|_{x=1}=0 \\
& \varphi(x, 0)=x, \quad-\left.D_{o}^{*} \frac{\partial \varphi}{\partial x}\right|_{x=0}+\varphi(0, t)=0,\left.\quad \frac{\partial \varphi}{\partial x}\right|_{x=1}=0
\end{aligned}
$$

Analytical Solution: Equations (9) - (13) satisfies (14) were solved analytically using parameter expanding method and Eigen function expansion techniques and we obtained

$$
\begin{aligned}
h(x, t)= & 1+\sum_{n=1}^{\infty} q_{0} e^{-q_{1} t} \sin \frac{(2 n-1) \pi x}{2}+\sum_{n=1}^{\infty} h_{n}(t) \sin \frac{(2 n-1) \pi x}{2} \\
\phi(x, t)= & e^{p_{10} t}+\sum_{n=1}^{\infty} p_{8} e^{p_{11} t} \cos n \pi x+\frac{f_{o}}{D_{b}^{*}}\left(e^{p_{10} t}+\sum_{n=1}^{\infty} p_{8} e^{p_{11} t}\right)\left(x-\frac{x^{2}}{2}\right)+ \\
& L_{o}(t)+\sum_{n=1}^{\infty} L_{n}(t) \cos n \pi x
\end{aligned}
$$




$$
\begin{gathered}
\psi(x, t)=e^{p_{10} t}+\sum_{n=1}^{\infty} p_{8} e^{p_{11} t} \cos n \pi x+\frac{f_{o}}{D_{v}^{*}}\left(e^{p_{7} t}+\sum_{n=1}^{\infty} p_{8} e^{p_{9} t}\right)\left(x-\frac{x^{2}}{2}\right)+ \\
Q_{o}(t)+\sum_{n=1}^{\infty} Q_{n}(t) \cos n \pi x \\
\theta(x, t)=p_{2}-p_{3} e^{-\lambda_{3} t}+\sum_{n=1}^{\infty}\left(p_{4} e^{-\lambda_{3} t}+p_{5} e^{-p_{6} t}\right) \cos n \pi x+
\end{gathered}
$$$$
\frac{f_{o}}{D_{c}^{*}}\left(p_{2}-p_{3} e^{-\lambda_{3} t}+\sum_{n=1}^{\infty}\left(p_{4} e^{-\lambda_{3} t}-p_{5} e^{-p_{6} t}\right)\right)\left(x-\frac{x^{2}}{2}\right)+N_{o}(t)+\sum_{n=1}^{\infty} N_{n}(t) \cos n \pi x
$$

Where,

$$
\begin{gathered}
\varphi(x, t)=e^{p_{10} t}+\sum_{n=1}^{\infty} p_{8} e^{p_{11} t} \cos n \pi x+\frac{f_{o}}{D_{o}^{*}}\left(1+\sum_{n=1}^{\infty} p e^{-p_{1} t}\right)\left(x-\frac{x^{2}}{2}\right)+ \\
M_{o}(t)+\sum_{n=1}^{\infty} M_{n}(t) \cos n \pi x
\end{gathered}
$$

$$
\begin{aligned}
& h_{n}(t)=\left(\begin{array}{l}
2 b_{3} e^{-p_{12} t}\left(\begin{array}{l}
\frac{2}{(2 n-1) \pi}\left(\frac{p_{2}}{p_{12}}\left(e^{p_{12} t}-1\right)-\frac{p_{3}}{p_{12}-\lambda_{3}}\left(e^{\left(p_{12}-\lambda_{3}\right) t}-1\right)\right)- \\
\sum_{n=1}^{\infty} \frac{2(2 n-1)}{(4 n-1) \pi}\left(\frac{p_{4}}{p_{12}-\lambda_{3}}\left(e^{\left(p_{12}-\lambda_{3}\right) t}-1\right)+\frac{p_{5}}{p_{12}-p_{6}}\left(e^{\left(p_{12}-p_{6}\right) t}-1\right)\right)
\end{array}\right)+\frac{4 b_{2} e^{-p_{12} t}}{p_{12}-\lambda_{3}}\left(\frac{\left(2(-1)^{n}+\pi-2 n \pi\right)}{\pi^{2}\left(4 n^{2}-4 n+1\right)}\right)\left(e^{\left(p_{12}-\lambda_{3}\right) t}-1\right)
\end{array}\right) \\
& M_{o}(t)=-\frac{2 f_{0} D_{4}}{R_{o} D_{o}^{*}}\left(t-\sum_{n=1}^{\infty} \frac{p\left(e^{-p_{1} t}-1\right)}{p_{1}}\right)-\frac{2 f_{0}}{3 D_{o}^{*}} \sum_{n=1}^{\infty} \frac{p p_{1}\left(e^{-p_{1} t}-1\right)}{p_{1}}- \\
& \sum_{n=1}^{\infty} \sum_{n=1}^{\infty} \frac{8 n(n \pi) p q_{0}(2 n-1)\left(e^{-\left(p_{1}+q_{1}\right) t}-1\right)}{R_{o}(4 n-1)\left(p_{1}+q_{1}\right)}+\frac{2 n_{1}}{3}- \\
& \frac{2 b_{16}}{R_{o}}\left(\begin{array}{l}
\frac{p_{2}\left(e^{p_{10} t}-1\right)}{p_{10}}-\frac{p_{3}\left(e^{\left(p_{10}+\lambda_{3}\right) t}-1\right)}{p_{10}-\lambda_{3}}+\sum_{n=1}^{\infty} \sum_{n=1}^{\infty} \frac{p p_{4}\left(e^{\left(p_{10}-p_{1}-\lambda_{3}\right) t}-1\right)}{2\left(p_{10}-p_{1}-\lambda_{3}\right)}+ \\
\sum_{n=1}^{\infty} \sum_{n=1}^{\infty} \frac{p p_{5}\left(e^{\left(p_{10}-p_{1}-p_{6}\right) t}-1\right)}{2\left(p_{10}-p_{1}-p_{6}\right)}+\sum_{n=1}^{\infty} \sum_{n=1}^{\infty} \frac{p p_{4}\left(e^{\left(p_{11}-\lambda_{3}\right) t}-1\right)}{2\left(p_{11}-\lambda_{3}\right)}+\sum_{n=1}^{\infty} \sum_{n=1}^{\infty} \frac{p p_{5}\left(e^{\left(p_{11}-p_{6}\right) t}-1\right)}{2\left(p_{11}-p_{6}\right)} \\
+\sum_{n=1}^{\infty} \sum_{n=1}^{\infty} \frac{p^{2} p_{2}\left(e^{\left(p_{11}-p_{1}\right) t}-1\right)}{2\left(p_{11}-p_{1}\right)}-\sum_{n=1}^{\infty} \sum_{n=1}^{\infty} \frac{p^{2} p_{3}\left(e^{\left(p_{11}-p_{1}-\lambda_{3}\right) t}-1\right)}{2\left(p_{11}-p_{1}-\lambda_{3}\right)}
\end{array}\right) \\
& M_{n}(t)=-\frac{2 f_{0} e^{-p_{1} t}}{(n \pi)^{2} D_{o}^{*}} \sum_{n=1}^{\infty} p p_{1} t+\frac{2 D_{4} b_{15} e^{-p_{1} t}}{R_{o}}\left(\sum_{n=1}^{\infty} \frac{p p_{2}(n \pi)^{2} t}{2}-\sum_{n=1}^{\infty} \frac{p p_{3}(n \pi)^{2}\left(e^{-\lambda_{3} t}-1\right)}{2 \lambda_{3}}\right)- \\
& \sum_{n=1}^{\infty} \sum_{n=1}^{\infty} \frac{8 n(n \pi) p q_{0}(2 n-1)\left(e^{-\left(p_{1}+q_{1}\right) t}-e^{-p_{1} t}\right)}{2 R_{o}\left(12 n^{2}+4 n-1\right) q_{1}}-\frac{2 n_{1} e^{-p_{1} t}}{(n \pi)^{2}}- \\
& \frac{2 b_{16} e^{-p_{1} t}}{R_{o}}\left(\begin{array}{l}
\sum_{n=1}^{\infty} \frac{p_{4}\left(e^{\left(p_{10}-\lambda_{3}\right) t}-1\right)}{2\left(p_{10}-\lambda_{3}\right)}+\sum_{n=1}^{\infty} \frac{p_{5}\left(e^{\left(p_{10}-p_{6}\right) t}-1\right)}{2\left(p_{10}-p_{6}\right)}+\sum_{n=1}^{\infty} \frac{p p_{2}\left(e^{p_{10} t}-1\right)}{2 p_{10}} \\
-\sum_{n=1}^{\infty} \frac{p p_{3}\left(e^{\left(p_{10}-\lambda_{3}\right) t}-1\right)}{2\left(p_{10}-\lambda_{3}\right)}+\sum_{n=1}^{\infty} \frac{p p_{2}\left(e^{p_{11} t}-1\right)}{2 p_{11}}+\sum_{n=1}^{\infty} \frac{p p_{3}\left(e^{-\lambda_{3} t}-1\right)}{2 \lambda_{3}} \\
+\sum_{n=1}^{\infty} \sum_{n=1}^{\infty} \sum_{n=1}^{\infty} \frac{3 p^{2} p_{4}\left(e^{\left(p_{11}-\lambda_{3}\right) t}-1\right)}{8\left(p_{11}-\lambda_{3}\right)}+\sum_{n=1}^{\infty} \sum_{n=1}^{\infty} \sum_{n=1}^{\infty} \frac{3 p^{2} p_{5}\left(e^{\left(p_{11}-p_{6}\right) t}-1\right)}{8\left(p_{11}-p_{6}\right)}
\end{array}\right) \\
& L_{o}(t)=e^{p_{10} t}\left(\frac{2 f_{0} \alpha_{1}}{3 R_{b} D_{b}^{*}}-\frac{2 f_{0} D_{1}}{R_{b} D_{b}^{*}}\right)\left(t+\sum_{n=1}^{\infty} \frac{p_{8}\left(e^{\left(p_{11}-p_{10}\right) t}-1\right)}{p_{11}-p_{10}}\right)-\frac{2 f_{0} e^{p_{10} t}}{3 D_{b}}\left(p_{10} t+\sum_{n=1}^{\infty} \frac{p_{8} p_{11}\left(e^{\left(p_{11}-p_{10}\right) t}-1\right)}{p_{11}-p_{10}}\right) \\
& +\sum_{n=1}^{\infty} \sum_{n=1}^{\infty} \frac{8(n \pi)^{2}(2 n-1) q_{0} p_{8}\left(e^{\left(p_{11}-q_{1}\right) t}-e^{p_{10} t}\right)}{2 R_{b} \pi(2 n-1)\left(p_{11}-p_{10}-q_{1}\right)} \\
& +\frac{2 e_{1} e^{p_{10} t}}{R_{b}}\left(p_{2} t+\frac{p_{3}\left(e^{-\lambda_{3} t}-1\right)}{\lambda_{3}}+\sum_{n=}^{\infty} \sum_{n=1}^{\infty} \frac{p_{4} p_{8}\left(e^{\left(p_{11}-p_{10}-\lambda_{3}\right) t}-1\right)}{2\left(p_{11}-p_{10}-\lambda_{3}\right)}+\sum_{n=1}^{\infty} \sum_{n=1}^{\infty} \frac{p_{5} p_{8}\left(e^{\left(p_{11}-p_{10}-p_{6}\right) t}-1\right)}{2\left(p_{11}-p_{10}-p_{6}\right)}\right)
\end{aligned}
$$




$$
\begin{aligned}
& +\frac{2 e_{2} e^{p_{10} t}}{R_{v}}\left(\begin{array}{l}
\frac{p_{2}\left(e^{p_{10} t}-1\right)}{p_{10}}-\frac{p_{3}\left(e^{\left(p_{10}-\lambda_{3}\right) t}-1\right)}{p_{10}-\lambda_{3}}+\sum_{n=1}^{\infty} \sum_{n=1}^{\infty} \frac{p_{2} p_{8}^{2}\left(e^{\left(2 p_{11}-p_{10}\right) t}-1\right)}{2\left(2 p_{11}-p_{10}\right)}- \\
\sum_{n=1}^{\infty} \sum_{n=1}^{\infty} \frac{p_{3} p_{8}^{2}\left(e^{\left(2 p_{11}-p_{10}-\lambda_{3}\right) t}-1\right)}{2\left(2 p_{11}-p_{10}-\lambda_{3}\right)}+\sum_{n=1}^{\infty} \sum_{n=1}^{\infty} \frac{p_{4} p_{8}\left(e^{\left(p_{11}-\lambda_{3}\right) t}-1\right)}{2\left(p_{11}-\lambda_{3}\right)} \\
+\sum_{n=1}^{\infty} \sum_{n=1}^{\infty} \frac{p_{5} p_{8}\left(e^{\left(p_{11}+p_{7}-p_{10}-p_{6}\right) t}-1\right)}{2\left(p_{11}+p_{7}-p_{10}-p_{6}\right)}
\end{array}\right) \\
& +\frac{2 e_{3} e^{p_{10} t}}{R_{b}}\left(\begin{array}{l}
\frac{p_{2}\left(e^{p_{7} t}-1\right)}{p_{7}}-\frac{p_{3}\left(e^{\left(p_{7}-\lambda_{3}\right) t}-1\right)}{p_{7}-\lambda_{3}}+\sum_{n=1}^{\infty} \sum_{n=1}^{\infty} \frac{p_{4} p_{8}\left(e^{\left(p_{11}+p_{7}-p_{10}-\lambda_{3}\right) t}-1\right)}{2\left(p_{11}+p_{7}-p_{10}-\lambda_{3}\right)}+ \\
\sum_{n=1}^{\infty} \frac{p_{5} p_{8}\left(e^{\left(p_{11}+p_{7}-p_{10}-p_{6}\right) t}-1\right)}{2\left(p_{11}+p_{7}-p_{10}-p_{6}\right)}+\sum_{n=1}^{\infty} \sum_{n=1}^{\infty} \frac{p_{4} p_{8}\left(e^{\left(p_{9}-\lambda_{3}\right) t}-1\right)}{2\left(p_{9}-\lambda_{3}\right)} \\
+\sum_{n=1}^{\infty} \sum_{n=1}^{\infty} \frac{p_{5} p_{8}\left(e^{\left(p_{9}-p_{6}\right) t}-1\right)}{2\left(p_{9}-p_{6}\right)}+\sum_{n=1}^{\infty} \sum_{n=1}^{\infty} \frac{p_{2} p_{8}^{2}\left(e^{\left(p_{11}+p_{9}-p_{10}-\lambda_{3}\right) t}-1\right)}{2\left(p_{11}+p_{9}-p_{10}-\lambda_{3}\right)}- \\
\sum_{n=1}^{\infty} \sum_{n=1}^{\infty} \frac{p_{3} p_{8}^{2}\left(e^{\left(p_{11}+p_{9}-p_{10}-p_{6}\right) t}-1\right)}{2\left(p_{11}+p_{9}-p_{10}-p_{6}\right)}
\end{array}\right) \\
& +\frac{2 b_{5} \alpha_{6} e^{p_{10} t}}{R_{b}}\left(\begin{array}{l}
p_{2} t+\frac{p_{3}\left(e^{-\lambda_{3} t}-1\right)}{\lambda_{3}}-\sum_{n=}^{\infty} \sum_{n=1}^{\infty} \frac{p_{4} p_{8}\left(e^{-\left(p_{1}+\lambda_{3}\right) t}-1\right)}{2\left(p_{1}+\lambda_{3}\right)}-\sum_{n=}^{\infty} \sum_{n=1}^{\infty} \frac{p_{5} p_{8}\left(e^{-\left(p_{1}+p_{6}\right) t}-1\right)}{2\left(p_{1}+p_{6}\right)} \\
+\sum_{n=1}^{\infty} \sum_{n=1}^{\infty} \frac{p_{4} p_{8}\left(e^{\left(p_{11}-p_{10}-\lambda_{3}\right) t}-1\right)}{2\left(p_{11}-p_{10}-\lambda_{3}\right)}+\sum_{n=}^{\infty} \sum_{n=1}^{\infty} \frac{p_{5} p_{8}\left(e^{\left(p_{11}-p_{10}-p_{6}\right) t}-1\right)}{2\left(p_{11}-p_{10}-p_{6}\right)} \\
+\sum_{n=1}^{\infty} \sum_{n=1}^{\infty} \frac{p_{2} p_{8}^{2}\left(e^{\left(p_{11}-p_{10}-p_{1}\right) t}-1\right)}{2\left(p_{11}-p_{10}-p_{1}\right)}-\sum_{n=}^{\infty} \sum_{n=1}^{\infty} \frac{p_{3} p_{8}^{2}\left(e^{\left(p_{11}-p_{10}-p_{1}-\lambda_{3}\right) t}-1\right)}{2\left(p_{11}-p_{10}-p_{1}-\lambda_{3}\right)}
\end{array}\right) \\
& -\frac{2 b_{7}\left(1-e^{p_{10} t}\right)}{R_{b} p_{10}}+\frac{2 n_{4} e^{p_{10} t}}{3} \\
& L_{n}(t)=-\frac{2 f_{0} \alpha_{1} e^{p_{11} t}}{R_{b} D_{b}^{*}(n \pi)^{2}}\left(\frac{e^{\left(p_{10}-p_{11}\right) t}}{p_{10}-p_{11}}+\sum_{n=1}^{\infty} p_{8} t\right)-\sum_{n=1}^{\infty} \sum_{n=1}^{\infty} \frac{8(n \pi)^{2}(2 n-1) q_{0} p_{8}\left(e^{\left(p_{11}-q_{1}\right) t}-e^{p_{11} t}\right)}{2 R_{b} \pi\left(12 n^{2}+4 n-1\right) q_{1}} \\
& -\frac{D_{1} b_{4} e^{p_{11} t}}{R_{b}}\left(\sum_{n=}^{\infty} \sum_{n=1}^{\infty} \frac{p_{3} p_{8}(n \pi)^{2}\left(e^{-\lambda_{3} t}-1\right)}{\lambda_{3}}+\sum_{n=1}^{\infty}(n \pi)^{2} p_{2} p_{8} t\right)+\frac{2 f_{0} e^{p_{10} t}}{D_{b}^{*}(n \pi)^{2}}\left(\sum_{n=1}^{\infty} p_{8} p_{11} t+\frac{p_{10}\left(e^{\left(p_{10}-p_{11}\right) t}-1\right)}{p_{10}-p_{11}}\right) \\
& +\frac{e_{1} e^{p_{11} t}}{R_{b}}\left(\sum_{n=1}^{\infty} p_{2} p_{8} t+\sum_{n=1}^{\infty} \frac{p_{3} p_{8}\left(e^{-\lambda_{3} t}-1\right)}{\lambda_{3}}+\sum_{n=1}^{\infty} \frac{p_{4}\left(e^{\left(p_{10}-p_{11}-\lambda_{3}\right) t}-1\right)}{\left(p_{10}-p_{11}-\lambda_{3}\right)}+\sum_{n=1}^{\infty} \frac{p_{5}\left(e^{\left(p_{10}-p_{11}-p_{6}\right) t}-1\right)}{\left(p_{10}-p_{11}-p_{6}\right)}\right) \\
& +\frac{2 e_{2} e^{p_{11} t}}{R_{b}}\left(\begin{array}{l}
\sum_{n=1}^{\infty} \frac{p_{2} p_{8}\left(e^{p_{7} t}-1\right)}{p_{7}}-\sum_{n=1}^{\infty} \frac{p_{3} p_{8}\left(e^{\left(p_{10}-\lambda_{3}\right) t}-1\right)}{p_{10}-\lambda_{3}}+\sum_{n=1}^{\infty} \frac{p_{4}\left(e^{\left(2 p_{10}-p_{11}-\lambda_{3}\right) t}-1\right)}{2\left(2 p_{10}-p_{11}-\lambda_{3}\right)}+ \\
\sum_{n=1}^{\infty} \frac{p_{4}\left(e^{\left(2 p_{10}-p_{11}-\lambda_{3}\right) t}-1\right)}{2\left(2 p_{10}-p_{11}-\lambda_{3}\right)}+\sum_{n=1}^{\infty} \frac{p_{5}\left(e^{\left(2 p_{10}-p_{11}-p_{6}\right) t}-1\right)}{2\left(2 p_{10}-p_{11}-p_{6}\right)}+\sum_{n=1}^{\infty} \sum_{n=1}^{\infty} \sum_{n=1}^{\infty} \frac{3 p_{4} p_{8}^{2}\left(e^{\left(p_{11}-\lambda_{3}\right) t}-1\right)}{8\left(p_{11}-\lambda_{3}\right)} \\
+\sum_{n=1}^{\infty} \sum_{n=1}^{\infty} \sum_{n=1}^{\infty} \frac{3 p_{5} p_{8}^{2}\left(e^{\left(p_{11}-p_{6}\right) t}-1\right)}{8\left(p_{11}-p_{6}\right)}
\end{array}\right) \\
& +\frac{2 e_{3} e^{p_{11} t}}{R_{b}}\left(\begin{array}{l}
\sum_{n=1}^{\infty} \frac{p_{2} p_{8}\left(e^{p_{7} t}-1\right)}{2 p_{7}}-\sum_{n=1}^{\infty} \frac{p_{3} p_{8}\left(e^{\left(p_{7}-\lambda_{3}\right) t}-1\right)}{2\left(p_{7}-\lambda_{3}\right)}+\sum_{n=1}^{\infty} \frac{p_{4}\left(e^{\left(p_{10}+p_{7}-p_{11}-\lambda_{3}\right) t}-1\right)}{2\left(p_{10}+p_{7}-p_{11}-\lambda_{3}\right)} \\
+\sum_{n=1}^{\infty} \frac{p_{5}\left(e^{\left(p_{10}+p_{7}-p_{11}-p_{6}\right) t}-1\right)}{2\left(p_{10}+p_{7}-p_{11}-p_{6}\right)}+\sum_{n=1}^{\infty} \frac{p_{2} p_{8}\left(e^{\left(p_{10}+p_{9}-p_{6}\right) t}-1\right)}{2\left(p_{10}+p_{9}-p_{6}\right)}- \\
\sum_{n=1}^{\infty} \frac{p_{3} p_{8}\left(e^{\left(p_{10}+p_{9}-p_{6}-\lambda_{3}\right) t}-1\right)}{2\left(p_{10}+p_{9}-p_{6}-\lambda_{3}\right)}+\sum_{n=1}^{\infty} \sum_{n=1}^{\infty} \sum_{n=1}^{\infty} \frac{3 p_{4} p_{8}^{2}\left(e^{\left(p_{9}-\lambda_{3}\right) t}-1\right)}{8\left(p_{9}-\lambda_{3}\right)} \\
+\sum_{n=1}^{\infty} \sum_{n=1}^{\infty} \sum_{n=1}^{\infty} \frac{3 p_{5} p_{8}^{2}\left(e^{\left(p_{9}-p_{6}\right) t}-1\right)}{8\left(p_{9}-p_{6}\right)}
\end{array}\right)+
\end{aligned}
$$




$$
\begin{aligned}
& \frac{2 b_{5} \alpha_{6} e^{p_{11} t}}{R_{b}}\left(\begin{array}{l}
\sum_{n=1}^{\infty} \frac{p_{4}\left(e^{\left(p_{10}-p_{11}-\lambda_{3}\right) t}-1\right)}{2\left(p_{10}-p_{11}-\lambda_{3}\right)}+\sum_{n=1}^{\infty} \frac{p_{5}\left(e^{\left(p_{10}-p_{11}-p_{6}\right) t}-1\right)}{2\left(p_{10}-p_{11}-p_{6}\right)}-\sum_{n=1}^{\infty} \frac{p_{3} p_{8}\left(e^{\left(p_{1}-p_{11}-\lambda_{3}\right) t}-1\right)}{2\left(p_{1}-p_{11}-\lambda_{3}\right)} \\
+\sum_{n=1}^{\infty} \frac{p_{2} p_{8}\left(e^{\left(p_{10}-p_{11}-p_{1}\right) t}-1\right)}{2\left(p_{10}-p_{11}-p_{1}\right)}-\sum_{n=1}^{\infty} \frac{p_{3} p_{8}\left(e^{\left(p_{10}-p_{11}-p_{1}-\lambda_{3}\right) t}-1\right)}{2\left(p_{10}-p_{11}-p_{1}-\lambda_{3}\right)}+\sum_{n=1}^{\infty} \frac{p_{2} p_{8} t}{2} \\
-\sum_{n=1}^{\infty} \sum_{n=}^{\infty} \sum_{n=1}^{\infty} \frac{3 p_{4} p_{8}^{2}\left(e^{-\left(p_{1}+\lambda_{3}\right) t}-1\right)}{8\left(p_{1}+\lambda_{3}\right)}-\sum_{n=1}^{\infty} \sum_{n=}^{\infty} \sum_{n=1}^{\infty} \frac{3 p_{5} p_{8}^{2}\left(e^{-\left(p_{1}+p_{6}\right) t}-1\right)}{8\left(p_{1}+p_{6}\right)}
\end{array}\right) \\
& -\sum_{n=1}^{\infty} \frac{b_{7} p_{8}\left(e^{-\left(p_{1}+2 p_{11}\right) t}-e^{p_{11} t}\right)}{R_{b}\left(p_{11}+p_{1}\right)}-\frac{2 n_{4} e^{p_{11} t}}{(n \pi)^{2}} \\
& N_{o}(t)=-\frac{2 f_{0} D_{3}}{R_{c} D_{c}^{*}}\left(p_{2} t+\frac{p_{3}\left(e^{-\lambda_{3} t}-1\right)}{\lambda_{3}}-\sum_{n=1}^{\infty}\left(\frac{p_{4}\left(e^{-\lambda_{3} t}-1\right)}{\lambda_{3}}+\frac{p_{5}\left(e^{-p_{6} t}-1\right)}{p_{6}}\right)\right)- \\
& \frac{2 f_{0}}{3 D_{c}^{*}}\left(-p_{3}\left(e^{-\lambda_{3} t}-1\right)+\sum_{n=1}^{\infty}\left(p_{4}\left(e^{-\lambda_{3} t}-1\right)+p_{5}\left(e^{-p_{6} t}-1\right)\right)\right)- \\
& \sum_{n=1}^{\infty} \sum_{n=1}^{\infty} \frac{8 n(n \pi) q_{0}(2 n-1)}{R_{o}(4 n-1)}\left(\frac{p_{4}\left(e^{-\left(p_{1}+q_{1}\right) t}-1\right)}{2\left(q_{1}+\lambda_{3}\right)}+\frac{p_{5}\left(e^{-\left(q_{1}+p_{6}\right) t}-1\right)}{2\left(q_{1}+p_{6}\right)}\right)+\frac{2 n_{2}}{3}- \\
& \frac{2 b_{12}}{R_{c}}\left(\frac{p_{2}\left(e^{p_{10} t}-1\right)}{p_{10}}-\frac{p_{3}\left(e^{\left(p_{10}-\lambda_{3}\right) t}-1\right)}{p_{10}-\lambda_{3}}+\sum_{n=1}^{\infty} \sum_{n=1}^{\infty} \frac{p p_{4}\left(e^{\left(p_{11}-\lambda_{3}\right) t}-1\right)}{2\left(p_{11}-\lambda_{3}\right)}+\sum_{n=1}^{\infty} \sum_{n=1}^{\infty} \frac{p p_{5}\left(e^{\left(p_{11}-p_{6}\right) t}-1\right)}{2\left(p_{11}-p_{6}\right)}\right)- \\
& \frac{2 b_{13}}{R_{c}}\left(\frac{p_{2}\left(e^{p_{7} t}-1\right)}{p_{7}}-\frac{p_{3}\left(e^{\left(p_{7}-\lambda_{3}\right) t}-1\right)}{p_{7}-\lambda_{3}}+\sum_{n=1}^{\infty} \sum_{n=1}^{\infty} \frac{p p_{4}\left(e^{\left(p_{9}-\lambda_{3}\right) t}-1\right)}{2\left(p_{9}-\lambda_{3}\right)}+\sum_{n=1}^{\infty} \sum_{n=1}^{\infty} \frac{p p_{5}\left(e^{\left(p_{9}-p_{6}\right) t}-1\right)}{2\left(p_{9}-p_{6}\right)}\right) \\
& N_{n}(t)=-\frac{2 f_{0} e^{-p_{6} t}}{(n \pi)^{2} D_{c}}\left(\frac{p_{3} \lambda_{3}\left(e^{\left(p_{6}-\lambda_{3}\right) t}-1\right)}{\left(p_{6}-\lambda_{3}\right)}-\sum_{n=1}^{\infty}\left(\frac{p_{3} \lambda_{3}\left(e^{\left(p_{6}-\lambda_{3}\right) t}-1\right)}{\left(p_{6}-\lambda_{3}\right)}+p_{5} p_{6} t\right)\right)+ \\
& \frac{D_{3} b_{11}}{R_{c}}\left(\sum_{n=1}^{\infty}(n \pi)^{2} e^{-p_{6} t}\left(\frac{p_{3} p_{4}\left(e^{\left(p_{6}-2 \lambda_{3}\right) t}-1\right)}{\left(p_{6}-2 \lambda_{3}\right)}-\frac{p_{3} p_{5}\left(e^{-\lambda_{3} t}-1\right)}{\lambda_{3}}-\frac{p_{2} p_{4}\left(e^{\left(p_{6}-\lambda_{3}\right) t}-1\right)}{\left(p_{6}-\lambda_{3}\right)}-p_{2} p_{5} t\right)\right)- \\
& \sum_{n=1}^{\infty} \sum_{n=1}^{\infty} \frac{8 n(n \pi) q_{0}(2 n-1) e^{-p_{6} t}}{R_{c}\left(12 n^{2}+4 n-1\right)}\left(\frac{p_{5}\left(e^{-q_{1} t}-1\right)}{2 q_{1}}-\frac{p_{4}\left(e^{\left(p_{6}-\lambda_{3}-q_{1}\right) t}-1\right)}{2\left(p_{6}-\lambda_{3}-q_{1}\right)}\right)-\frac{2 n_{2} e^{-p_{1} t}}{(n \pi)^{2}}- \\
& \frac{b_{12} e^{-p_{6} t}}{R_{c}}\left(\sum_{n=1}^{\infty} \frac{p p_{2}\left(e^{\left(p_{6}+p_{1}\right) t}-1\right)}{p_{6}-p_{11}}-\sum_{n=1}^{\infty} \frac{p p_{3}\left(e^{\left(p_{6}+p_{11}-\lambda_{3}\right) t}-1\right)}{\left(p_{6}+p_{11}-\lambda_{3}\right)}+\sum_{n=1}^{\infty} \frac{p_{4}\left(e^{\left(p_{10}+p_{6}-\lambda_{3}\right) t}-1\right)}{\left(p_{10}+p_{6}-\lambda_{3}\right)}+\sum_{n=1}^{\infty} \frac{p_{5}\left(e^{p_{10} t}-1\right)}{p_{10}}\right)- \\
& \frac{b_{13} e^{-p_{6} t}}{R_{c}}\left(\sum_{n=1}^{\infty} \frac{p p_{2}\left(e^{\left(p_{6}+p_{9}\right) t}-1\right)}{p_{6}+p_{2}}-\sum_{n=1}^{\infty} \frac{p p_{3}\left(e^{\left(p_{6}+p_{9}-\lambda_{3}\right) t}-1\right)}{\left(p_{6}+p_{9}-\lambda_{3}\right)}+\sum_{n=1}^{\infty} \frac{p_{4}\left(e^{\left(p_{6}+p_{7}-\lambda_{3}\right) t}-1\right)}{\left(p_{6}+p_{7}-\lambda_{3}\right)}+\sum_{n=1}^{\infty} \frac{p_{5}\left(e^{p_{7} t}-1\right)}{p_{7}}\right) \\
& Q_{o}(t)=e^{p_{7} t}\left(\frac{2 f_{0} r_{0}}{3 R_{v} D_{v}^{*}}-\frac{2 f_{0} D_{2}}{R_{v} D_{v}^{*}}\right)\left(t+\sum_{n=1}^{\infty} \frac{p_{8}\left(e^{\left(p_{9}-p_{7}\right) t}-1\right)}{p_{9}-p_{7}}\right)-\frac{2 f_{0} e^{p_{7} t}}{3 D_{v}^{*}}\left(p_{7} t+\sum_{n=1}^{\infty} \frac{p_{8} p_{9}\left(e^{\left(p_{9}-p_{7}\right) t}-1\right)}{p_{9}-p_{7}}\right) \\
& +\sum_{n=1}^{\infty} \sum_{n=1}^{\infty} \frac{8(n \pi)^{2}(2 n-1) q_{0} p_{8}\left(e^{\left(p_{9}-q_{1}\right) t}-e^{p_{7} t}\right)}{2 R_{v} \pi(2 n-1)\left(p_{9}-p_{7}-q_{1}\right)} \\
& +\frac{2 e_{4} e^{p_{7} t}}{R_{v}}\left(p_{2} t+\frac{p_{3}\left(e^{-\lambda_{3} t}-1\right)}{\lambda_{3}}+\sum_{n=}^{\infty} \sum_{n=1}^{\infty} \frac{p p_{4}\left(e^{\left(p_{9}-p_{7}-\lambda_{3}\right) t}-1\right)}{2\left(p_{9}-p_{7}-\lambda_{3}\right)}+\sum_{n=}^{\infty} \sum_{n=1}^{\infty} \frac{p p_{5}\left(e^{\left(p_{9}-p_{7}-p_{6}\right) t}-1\right)}{2\left(p_{9}-p_{7}-p_{6}\right)}\right) \\
& +\frac{2 e_{5} e^{p_{7} t}}{R_{v}}\left(\begin{array}{l}
\frac{p_{2}\left(e^{p_{10} t}-1\right)}{p_{10}}-\frac{p_{3}\left(e^{\left(p_{10}-\lambda_{3}\right) t}-1\right)}{p_{10}-\lambda_{3}}+\sum_{n=1}^{\infty} \sum_{n=1}^{\infty} \frac{p_{4} p_{8}\left(e^{\left(p_{10}+p_{9}-p_{7}-\lambda_{3}\right) t}-1\right)}{2\left(p_{10}+p_{9}-p_{7}-\lambda_{3}\right)}+ \\
\sum_{n=1}^{\infty} \sum_{n=1}^{\infty} \frac{p_{5} p_{8}\left(e^{\left(p_{10}+p_{9}-p_{7}-p_{6}\right) t}-1\right)}{2\left(p_{10}+p_{9}-p_{7}-p_{6}\right)}+\sum_{n=1}^{\infty} \sum_{n=1}^{\infty} \frac{p_{4} p_{8}\left(e^{\left(p_{11}-\lambda_{3}\right) t}-1\right)}{2\left(p_{11}-\lambda_{3}\right)} \\
+\sum_{n=1}^{\infty} \sum_{n=1}^{\infty} \frac{p_{5} p_{8}\left(e^{\left(p_{11}-p_{6}\right) t}-1\right)}{2\left(p_{11}-p_{6}\right)}+\sum_{n=1}^{\infty} \sum_{n=1}^{\infty} \frac{p_{2} p_{8}^{2}\left(e^{\left(p_{11}+p_{9}-p_{7}\right) t}-1\right)}{2\left(p_{11}+p_{9}-p_{7}\right)}+ \\
-\sum_{n=1}^{\infty} \sum_{n=1}^{\infty} \frac{p_{3} p_{8}^{2}\left(e^{\left(p_{11}+p_{9}-p_{7}-\lambda_{3}\right) t}-1\right)}{2\left(p_{11}+p_{9}-p_{7}-\lambda_{3}\right)}
\end{array}\right)
\end{aligned}
$$




$$
\begin{aligned}
& +\frac{2 e_{6} e^{p_{9} t}}{R_{v}}\left(\begin{array}{l}
\sum_{n=1}^{\infty} \frac{p_{2} p_{8}\left(e^{p_{7} t}-1\right)}{p_{7}}-\sum_{n=1}^{\infty} \frac{p_{3} p_{8}\left(e^{\left(p_{7}-\lambda_{3}\right) t}-1\right)}{p_{7}-\lambda_{3}}+\sum_{n=1}^{\infty} \frac{p_{4}\left(e^{\left(2 p_{7}-p_{9}-\lambda_{3}\right) t}-1\right)}{2\left(2 p_{7}-p_{9}-\lambda_{3}\right)} \\
+\sum_{n=1}^{\infty} \frac{p_{5}\left(e^{\left(2 p_{7}-p_{9}-p_{6}\right) t}-1\right)}{2\left(2 p_{7}-p_{9}-p_{6}\right)}+\sum_{n=1}^{\infty} \sum_{n=1}^{\infty} \sum_{n=1}^{\infty} \frac{3 p_{4} p_{8}^{2}\left(e^{\left(p_{9}-\lambda_{3}\right) t}-1\right)}{8\left(p_{9}-\lambda_{3}\right)} \\
+\sum_{n=1}^{\infty} \sum_{n=1}^{\infty} \sum_{n=1}^{\infty} \frac{3 p_{5} p_{8}^{2}\left(e^{\left(p_{9}-p_{6}\right) t}-1\right)}{8\left(p_{9}-p_{6}\right)}
\end{array}\right) \\
& +\frac{2 b_{9} \alpha_{6} e^{p_{9} t}}{R_{v}}\left(\begin{array}{l}
\sum_{n=1}^{\infty} \frac{p_{4}\left(e^{\left(p_{7}-p_{9}-\lambda_{3}\right) t}-1\right)}{2\left(p_{7}-p_{9}-\lambda_{3}\right)}+\sum_{n=1}^{\infty} \frac{p_{5}\left(e^{\left(p_{7}-p_{9}-p_{6}\right) t}-1\right)}{2\left(p_{7}-p_{9}-p_{6}\right)}+\sum_{n=1}^{\infty} \frac{p_{2} p_{8} t}{2}+\sum_{n=1}^{\infty} \frac{p_{3} p_{8}\left(e^{-\lambda_{3} t}-1\right)}{2 \lambda_{3}} \\
+\sum_{n=1}^{\infty} \frac{p_{2} p_{8}\left(e^{\left(p_{7}-p_{9}-p_{1}\right) t}-1\right)}{2\left(p_{7}-p_{9}-p_{1}\right)}-\sum_{n=1}^{\infty} \frac{p_{3} p_{8}\left(e^{\left(p_{7}-p_{9}-p_{1}-\lambda_{3}\right) t}-1\right)}{2\left(p_{7}-p_{9}-p_{1}-\lambda_{3}\right)} \\
-\sum_{n=1}^{\infty} \sum_{n=1}^{\infty} \sum_{n=1}^{\infty} \frac{3 p_{4} p_{8}^{2}\left(e^{-\left(p_{1}+\lambda_{3}\right) t}-1\right)}{8\left(p_{1}+\lambda_{3}\right)}-\sum_{n=1}^{\infty} \sum_{n=}^{\infty} \sum_{n=1}^{\infty} \frac{3 p_{5} p_{8}^{2}\left(e^{-\left(p_{1}+p_{6}\right) t}-1\right)}{8\left(p_{1}+p_{6}\right)}
\end{array}\right) \\
& -\frac{2 n_{3} e^{p_{9} t}}{(n \pi)^{2}}
\end{aligned}
$$$$
Q_{n}(t)=-\frac{2 f_{0} r_{0} e^{p_{9} t}}{R_{v} D_{v}^{*}(n \pi)^{2}}\left(\frac{e^{\left(p_{9}-p_{7}\right) t}}{p_{9}-p_{7}}+\sum_{n=1}^{\infty} p_{8} t\right)+\frac{2 f_{0} e^{p_{9} t}}{D_{v}^{*}(n \pi)^{2}}\left(\sum_{n=1}^{\infty} p_{8} p_{9} t+\frac{p_{7}\left(e^{\left(p_{7}-p_{9}\right) t}-1\right)}{p_{7}-p_{9}}\right)
$$$$
+\frac{D_{2} b_{8} e^{p_{9} t}}{R_{v}}\left(-\sum_{n=1}^{\infty} \sum_{n=1}^{\infty} \frac{p_{3} p_{8}(n \pi)^{2}\left(e^{-\lambda_{3} t}-1\right)}{\lambda_{3}}-\sum_{n=1}^{\infty}(n \pi)^{2} p_{2} p_{8} t\right)
$$$$
-\sum_{n=1}^{\infty} \sum_{n=1}^{\infty} \frac{8(n \pi)^{2}(2 n-1) q_{0} p_{8}\left(e^{\left(p_{9}-q_{1}\right) t}-e^{p_{9} t}\right)}{2 R_{v} \pi\left(12 n^{2}+4 n-1\right) q_{1}}
$$$$
+\frac{e_{4} e^{p_{9} t}}{R_{v}}\left(\sum_{n=1}^{\infty} p_{2} p t+\sum_{n=1}^{\infty} \frac{p p_{3}\left(e^{-\lambda_{3} t}-1\right)}{\lambda_{3}}+\sum_{n=1}^{\infty} \frac{p_{4}\left(e^{\left(p_{7}-p_{9}-\lambda_{3}\right) t}-1\right)}{\left(p_{7}-p_{9}-\lambda_{3}\right)}+\sum_{n=1}^{\infty} \frac{p_{5}\left(e^{\left(p_{7}-p_{9}-p_{6}\right) t}-1\right)}{\left(p_{7}-p_{9}-p_{6}\right)}\right)
$$$$
\left(\frac{p_{2}\left(e^{p_{10} t}-1\right)}{p_{10}}-\frac{p_{3}\left(e^{\left(p_{10}-\lambda_{3}\right) t}-1\right)}{p_{10}-\lambda_{3}}+\sum_{n=1}^{\infty} \sum_{n=1}^{\infty} \frac{p_{4} p_{8}\left(e^{\left(p_{10}+p_{9}-p_{7}-\lambda_{3}\right) t}-1\right)}{2\left(p_{10}+p_{9}-p_{7}-\lambda_{3}\right)}+\right.
$$$$
\left.+\frac{2 e_{5} e^{p_{9} t}}{R_{v}} \mid \begin{array}{l}
\sum_{n=1}^{\infty} \frac{p_{4}\left(e^{\left(p_{10}-p_{9}+p_{7}-\lambda_{3}\right) t}-1\right)}{2\left(p_{10}-p_{9}+p_{7}-\lambda_{3}\right)}+\sum_{n=1}^{\infty} \frac{p_{5}\left(e^{\left(p_{10}-p_{9}+p_{7}-p_{6}\right) t}-1\right)}{2\left(p_{10}-p_{9}+p_{7}-p_{6}\right)} \\
+\sum_{n=1}^{\infty} \frac{p_{2} p_{8}\left(e^{p_{10} t}-1\right)}{2 p_{10}}-\sum_{n=1}^{\infty} \frac{p_{3} p_{8}\left(e^{\left(p_{10}-\lambda_{3}\right) t}-1\right)}{2\left(p_{10}-\lambda_{3}\right)}+\sum_{n=1}^{\infty} \frac{p_{2} p_{8}\left(e^{\left(p_{11}+p_{7}-p_{9}\right) t}-1\right)}{2\left(p_{11}+p_{7}-p_{9}\right)} \\
-\sum_{n=1}^{\infty} \frac{p_{3} p_{8}\left(e^{\left(p_{11}+p_{7}-p_{9}-\lambda_{3}\right) t}-1\right)}{2\left(p_{11}+p_{7}-p_{9}-\lambda_{3}\right)}+\sum_{n=1}^{\infty} \sum_{n=1}^{\infty} \sum_{n=1}^{\infty} \frac{p_{4} p_{8}^{2}\left(e^{\left(p_{11}-\lambda_{3}\right) t}-1\right)}{2\left(p_{11}-\lambda_{3}\right)}+ \\
\sum_{n=1}^{\infty} \sum_{n=1}^{\infty} \sum_{n=1}^{\infty} \frac{3 p_{5} p_{8}^{2}\left(e^{\left(p_{11}-p_{6}\right) t}-1\right)}{8\left(p_{11}-p_{6}\right)}
\end{array}\right)-
$$$$
+\frac{2 e_{6} e^{p_{9} t}}{R_{v}}\left(\begin{array}{l}
\sum_{n=1}^{\infty} \frac{p_{2} p_{8}\left(e^{p_{7} t}-1\right)}{p_{7}}-\sum_{n=1}^{\infty} \frac{p_{3} p_{8}\left(e^{\left(p_{7}-\lambda_{3}\right) t}-1\right)}{p_{7}-\lambda_{3}}+\sum_{n=1}^{\infty} \frac{p_{4}\left(e^{\left(2 p_{7}-p_{9}-\lambda_{3}\right) t}-1\right)}{2\left(2 p_{7}-p_{9}-\lambda_{3}\right)} \\
+\sum_{n=1}^{\infty} \frac{p_{5}\left(e^{\left(2 p_{7}-p_{9}-p_{6}\right) t}-1\right)}{2\left(2 p_{7}-p_{9}-p_{6}\right)}+\sum_{n=1}^{\infty} \sum_{n=1}^{\infty} \sum_{n=1}^{\infty} \frac{3 p_{4} p_{8}^{2}\left(e^{\left(p_{9}-\lambda_{3}\right) t}-1\right)}{8\left(p_{9}-\lambda_{3}\right)} \\
+\sum_{n=1}^{\infty} \sum_{n=1}^{\infty} \sum_{n=1}^{\infty} \frac{3 p_{5} p_{8}^{2}\left(e^{\left(p_{9}-p_{6}\right) t}-1\right)}{8\left(p_{9}-p_{6}\right)}
\end{array}\right)
$$$$
+\frac{2 b_{9} \alpha_{6} e^{p_{9} t}}{R_{v}}\left(\begin{array}{l}
\sum_{n=1}^{\infty} \frac{p_{4}\left(e^{\left(p_{7}-p_{9}-\lambda_{3}\right) t}-1\right)}{2\left(p_{7}-p_{9}-\lambda_{3}\right)}+\sum_{n=1}^{\infty} \frac{p_{5}\left(e^{\left(p_{7}-p_{9}-p_{6}\right) t}-1\right)}{2\left(p_{7}-p_{9}-p_{6}\right)}+\sum_{n=1}^{\infty} \frac{p_{2} p_{8} t}{2}+\sum_{n=1}^{\infty} \frac{p_{3} p_{8}\left(e^{-\lambda_{3} t}-1\right)}{2 \lambda_{3}} \\
+\sum_{n=1}^{\infty} \frac{p_{2} p_{8}\left(e^{\left(p_{7}-p_{9}-p_{1}\right) t}-1\right)}{2\left(p_{7}-p_{9}-p_{1}\right)}-\sum_{n=1}^{\infty} \frac{p_{3} p_{8}\left(e^{\left(p_{7}-p_{9}-p_{1}-\lambda_{3}\right) t}-1\right)}{2\left(p_{7}-p_{9}-p_{1}-\lambda_{3}\right)} \\
-\sum_{n=1}^{\infty} \sum_{n=1}^{\infty} \sum_{n=1}^{\infty} \frac{3 p_{4} p_{8}^{2}\left(e^{-\left(p_{1}+\lambda_{3}\right) t}-1\right)}{8\left(p_{1}+\lambda_{3}\right)}-\sum_{n=1}^{\infty} \sum_{n=1}^{\infty} \sum_{n=1}^{\infty} \frac{3 p_{5} p_{8}^{2}\left(e^{-\left(p_{1}+p_{6}\right) t}-1\right)}{8\left(p_{1}+p_{6}\right)}
\end{array}\right)-\frac{2 n_{3} e^{p_{9} t}}{(n \pi)^{2}}
$$ 


$$
\begin{aligned}
& b_{2}=\frac{\lambda_{1}}{\alpha_{0}}, \quad b_{3}=\frac{\lambda_{2}}{\alpha_{0}}, \quad b_{4}=\frac{\sigma_{1}}{\alpha_{0}}, \quad b_{5}=\frac{\alpha_{4}}{\alpha_{0}}, \quad b_{6}=\frac{\alpha_{9}}{\alpha_{0}}, \quad b_{7}=\frac{\alpha_{3}}{\alpha_{0}} b_{8}=\frac{\gamma_{1}}{\alpha_{0}}, \quad b_{9}=\frac{r_{1}}{\alpha_{0}}, \\
& b_{10}=\frac{r_{2}}{\alpha_{0}}, \quad b_{11}=\frac{v_{1}}{\alpha_{0}}, \quad b_{12}=\frac{m_{1}}{\alpha_{0}}, \quad b_{13}=\frac{m_{2}}{\alpha_{0}}, \quad b_{15}=\frac{\mu_{1}}{\alpha_{0}}, \quad b_{16}=\frac{g_{1}}{\alpha_{0}}, f_{0}=\frac{1}{\alpha_{0}}, \lambda_{1}=\frac{1}{\beta_{p} \lambda_{c}} \text {, } \\
& m_{3}=\frac{\alpha_{s}}{\beta_{p}}, \quad e_{1}=b_{5} \alpha_{5}+b_{6} \alpha_{5}, \quad e_{2}=b_{5} \alpha_{7}+b_{6} \beta_{1}, \quad e_{3}=b_{5} \alpha_{8}+b_{6} \beta_{2}, \quad e_{4}=b_{9} \alpha_{5}+b_{10} \alpha_{5}, \\
& e_{5}=b_{9} \alpha_{7}+b_{10} \beta_{1}, \quad e_{6}=b_{9} \alpha_{8}+b_{10} \beta_{2} \text {, } \\
& p=p_{8}=\frac{2\left((-1)^{n}-1\right)}{(n \pi)^{2}}, \quad p_{1}=\frac{D_{4}(n \pi)^{2}}{R_{o}}, \quad p_{2}=1+\frac{2 m_{3}}{\lambda_{3} R_{c}}, \quad p_{3}=\frac{2 m_{3}}{\lambda_{3} R_{c}}, \quad p_{4}=\frac{2 m_{3}\left((-1)^{n}-1\right)}{R_{c}(n \pi)^{2}\left(\frac{D_{3}(n \pi)^{2}}{R_{c}}-\lambda_{3}\right)}, \\
& p_{5}=\frac{2\left((-1)^{n}-1\right)}{(n \pi)^{2}}\left(1-\frac{m_{3}}{R_{c}\left(\frac{D_{3}(n \pi)^{2}}{R_{c}}-\lambda_{3}\right)}\right), \quad p_{6}=\frac{D_{3}(n \pi)^{2}}{R_{c}}, \quad p_{7}=\frac{r_{0}}{R_{v}}, \quad p_{9}=\frac{\left(r_{0}-D_{2}(n \pi)^{2}\right)}{R_{v}}, p_{10}=\frac{\alpha_{1}}{R_{b}}, \\
& p_{11}=\frac{\left(\alpha_{1}-D_{1}(n \pi)^{2}\right)}{R_{b}}, \quad p_{12}=\left(\frac{(2 n-1) \pi}{2}\right)^{2}, \quad n_{1}=-\frac{f_{0}\left(1+\sum_{n=1}^{\infty} p\right)}{D_{o}^{*}}, n_{2}=-\frac{f_{0}\left(p_{2}-p_{3}+\sum_{n=1}^{\infty}\left(p_{4}+p_{5}\right)\right)}{D_{c}^{*}}, \\
& n_{3}=-\frac{f_{0}\left(1+\sum_{n=1}^{\infty} p_{8}\right)}{D_{v}^{*}}, \quad n_{4}=-\frac{f_{0}\left(1+\sum_{n=1}^{\infty} p_{8}\right)}{D_{b}^{*}}
\end{aligned}
$$

\section{RESULTS AND DISCUSSION}

In this section, the governing equations (9) - (14) were solved analytically using parameter expanding method and Eigen functions expansions techniques, the solutions are discussed with the help of input data. From Figures 2 and 3 we observed that the hydraulic head $h$ increases along distance and decreases with time, but increases with increase in pumping rate $\beta_{p}$. Figure 4 shows a sinusoidal form along distance and later decrease, but increases with increase in Pumping rate $\beta_{p}$. In a similar manner in Figures 5 and 6 the pumping rate increases the concentration of contaminant $\theta$ along distance and later decreases.

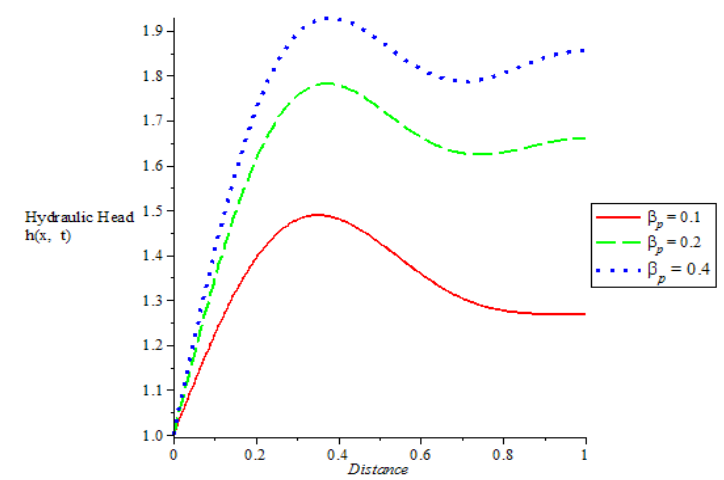

Fig 2: Relation between Hydraulic head against distance at various values of pumping rate.

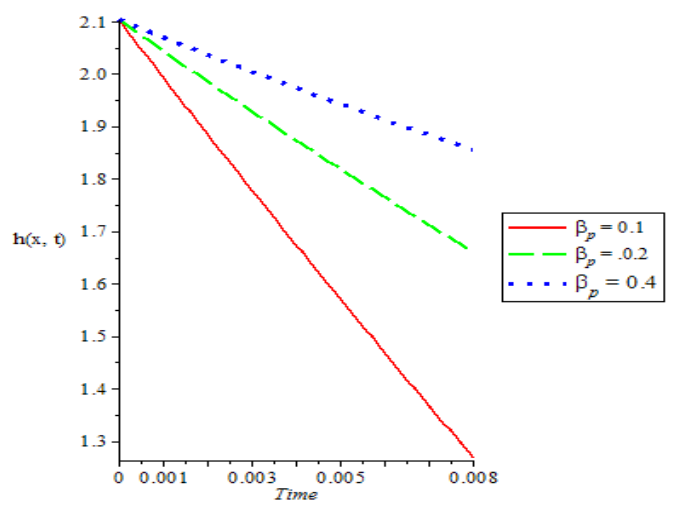

Fig 3: Relation between Hydraulic head against time at various

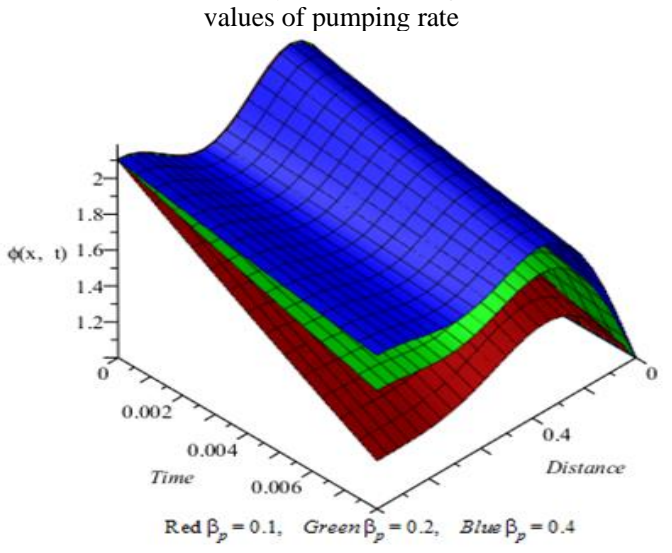

Fig 4: Relationships among bacteria concentration, distance and time at various values of pumping rate 


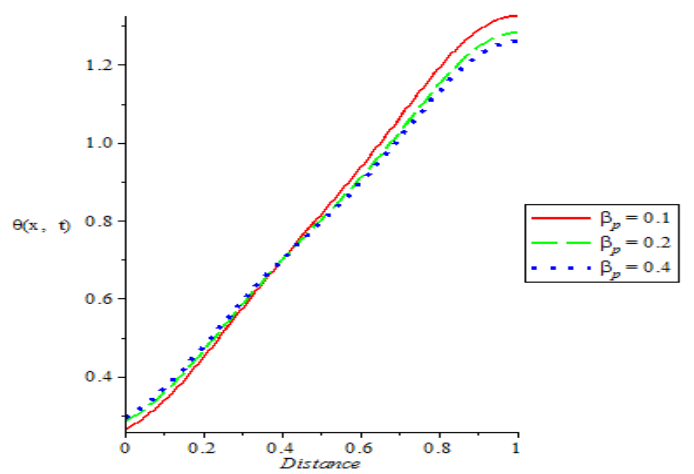

Fig 5: Relation between contaminant concentrations against distance at various values of pumping rate

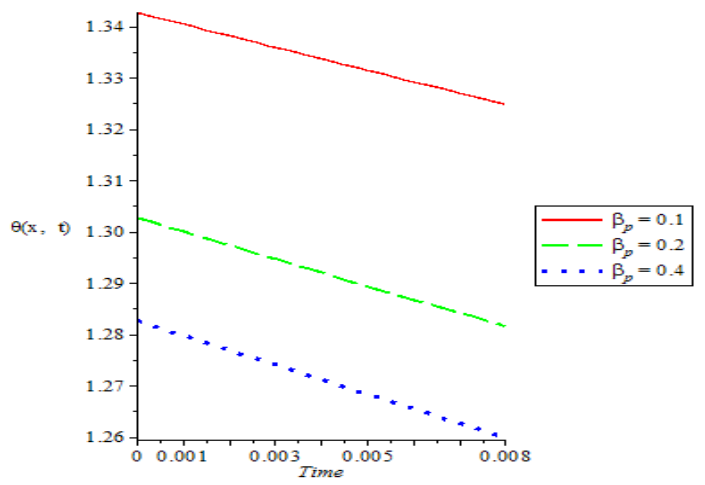

Fig 6: Relation between contaminant concentrations against time at various values of pumping rate

Conclusion: We developed unsteady one dimensional analytical model that incorporates variable dependent pumping rates on a contaminant transport in riverbank filtration system. Based on the above results we can conclude that: Pumping rate values increases both the hydraulic head and concentration of colloids. The quality of pumped water from RBF will slightly reduce due to the increase in pumping rate. The bacteria concentrations of the pumped water will be high if the pumping well is closed to the river.

\section{REFERENCE}

Aiyesimi, YM; Jimoh, OR (2012). Computational analysis of 1-dimensional non-linear reactive contaminant flow problem with an initial continuous point source. J. Nig. Assoc. Math. Physics. 22: 543 - 558.

Bohaienko, V; Bulavatsky, V (2019). Mathematical modeling of solutes migration under the conditions of groundwater filtration by the model with the kCaputo fractional derivative. Fractal and Fractional. 2(28): 1 -10.
Chattopadhyay, BB; Deo, SS (2018). Mathematical modeling of concentration of contaminant level for water in Damodar River Bermo Region. Global J. Eng. Sci. Res. 5(8): $35-44$.

Gothwal, R; Thatikonda, S (2017). Mathematical model for the transport of fluoroquinolone and its resistant bacteria in aquatic environment. Environ. Sci. Pollute. Res. 1 - 14, DOI 10.1007/s11356-0179848-x.

Grischek, T; Schoenheinz, D; Ray, C (2002). Siting and design issues for riverbank filtration schemes. Riverbank filtration: Improving source water quality. Springer. 291-302.

Kim, S; Corapcioglu, MY; Kim, D (2003). Effect of dissolved organic matter and bacteria on contaminant transport in riverbank filtration. Journal of Contaminant Hydrology. 66: 1- 23.

Lee, SI; Lee, SS (2010). Development of site suitability analysis system for riverbank filtration. Water Science Engineering. 3(1): 85-94.

Mustafa, S; Abdul Aziz, Z; Bahar, A; Shamsuddin, MKN (2018). Modelling the effect of hydraulic conductivity on one dimensional contaminant transport in RBF system. Matemateka. 34(2): 261 -269 .

Mustafa, S; Bahar, A; Aziz, ZA; Suratman, S (2014). Review of the role of analytical modelling methods in riverbank filtration system. Jurnal Teknologi (Sciences and Engineering). 71(1): 59 - 69.

Ray, C (2011). Riverbank filtration concepts and applicability to desert environments. Riverbank filtration for water security in desert countries. Springer. 1-4.

Shamsuddin, MKN; Suratman, S; Zakaria, MP; Aris, AZ; Sulaiman, WNA (2014). Particle tracking analysis of river-aquifer interaction via bank infiltration techniques. Environ. Earth Sci.. DOI 10.1007/s12665-014-3217-6.

Singh, MK; Mahato, NK; Kumari, P (2011). Comparative study of analytical solutions for timedependent solute transport along unsteady groundwater flow in semi-infinite aquifer. Inter. $J$. Geosciences. 2(4): 457-467. 